\title{
Pattern of Hematological Manifestations in Patients with Systemic Lupus Erythematosus Attending in a Tertiary Care Hospital
}

\author{
Md Abdur Rouf ${ }^{*}$ \\ Abdul Ahad Mohammed Ryhan Uddin ${ }^{2}$ \\ Ehathesamul Hoque ${ }^{3}$ \\ Mohammad Ismail ${ }^{4}$ \\ Rajat Sanker Roy Biswas ${ }^{5}$ \\ Farzana Alam 6 \\ Mohammad Shirajul Islam
}

'Department of Medicine and Cardiology University of Science \& Technology Chittagong Chittagong, Bangladesh.

${ }^{2}$ Department of Medicine and Critical Care Unit University of Science \& Technology Chittagong Chittagong, Bangladesh.

${ }^{3}$ Department of Nephrology

Chattagram Maa-O-Shishu Hospital Medical College Chittagong, Bangladesh

${ }^{4}$ Department of Dermatology and Venereology University of Science \& Technology Chittagong Chittagong, Bangladesh.

${ }^{5}$ Department of Medicine

Chattagram International Dental College \& Hospital International Islamic Medical College \& Hospital Chittagong, Bangladesh.

${ }^{6}$ Department of Biochemistry Chittagong Medical College

Chittagong, Bangladesh.

${ }^{7}$ Department of Medicine Chittagong Medical College Chittagong, Bangladesh.

${ }^{*}$ Correspondence to:

\section{Dr. Md. Abdur Rouf}

Department of Medicine and Cardiology

University of Science \& Technology Chittagong

Chittagong, Bangladesh.

Mobile: 01937295552

E-mail:roufdr64@gmail.com

\begin{abstract}
Hematological abnormalities are frequently encountered in patients with SLE. It was aimed to study the pattern and frequency of hematological abnormalities in patients with SLE. Forty patients were prospectively analyzed who were suffering from SLE in the $\mathrm{CMCH}$ over a period of one year. All patients were evaluated according to the case record form with particular reference to hematological manifestations. Most patients were females in the child bearing age. Hematological manifestations were documented in $87.5 \%$ of patients. Anemia was the most common hematological abnormality detected (57.5\%). Iron deficiency anemia(IDA) $60 \%$, Anemia of chronic disease(ACD) $26.9 \%$ and Autoimmune hemolytic anemia(AIHA) $8.7 \%$ were the most common cause of anemia detected. The data demonstrated that while hematological manifestations are very common in SLE but they are rarely life threatening.
\end{abstract}

Key words: Systemic lupus erythematosus; thrombocytopenia; lymphopenia

\section{INTRODUCTION}

Systemic Lupus Erythematosus (SLE) is a multisystem autoimmune disease that predominantly affects women of child bearing age. It is also a major cause of mortality and morbidity in the young populations ${ }^{1}$.

The prevalence of SLE varies throughout the world. In the western world, it is about 40 per 100000 populations. There appear to be a higher incidence in blacks and Hispanics than in other population. Over 80 percent of cases occur in women during their childbearing age ${ }^{2}$.

The prognosis of SLE in western world has improved remarkably in the past few decades, from a 5 - year survival rate of only $50 \%$ in the 1990 s to 10 year survival rate of nearly $90 \%$ in the 1995 . However, a poor survival of SLE is still reported in certain ethnic groups such as Indians, Black Caribbeans and Hispanics. Severe organ involvement related to SLE itself and infection remain the main causes of mortality ${ }^{3}$.

Hematological abnormalities are frequently encountered in patients with SLE and are part of American Rheumatological Association Criteria for classifying the disease. But there is a relative paucity of information on the significance of these abnormalities in the course of the condition ${ }^{4}$.

Anemia is the most common hematological abnormality seen in SLE, Autoimmune hemolytic anemia occurs in approximately $5-10 \%$ of SLE patients and it responds well to corticosteroid treatments. Neutropenia is relatively common and has been related to an increased risk of infection in patients with SLE. Although thrombocytopenia caused by peripheral immune destruction is common in SLE(20$40 \%)$, severe thrombocytopenia is comparatively rare $(\sim 5 \%)$. It is now recognized that lymphopenia is one of the most common hematological findings in $\mathrm{SLE}^{5,6}$.

The objective of the present study is to document different hematological manifestations among the SLE patients and its pattern as there are scarcity of studies regarding these field in the context of Chittagong which is situated in the southern part of Bangladesh. 


\section{MATERIALS \& METHODS}

The study was carried out in 40 SLE patients. It was done from January 2010 to December 2010. All the samples were collected from the Medicine, Nephrology and Dermatology wards of a tertiary care medical college hospital of Chittagong. Adult patients of SLE as evidenced by American Rheumatological Criteria (ARA) were included in the study and patients with hematological problems for due to other diseases were excluded. A structured questionnaire and blood film study were used as research instrument. Detailed history was collected in the structured questionnaire which includes name, age, sex, occupation, monthly income, academic qualifications and co-morbidity. The form also included different physical signs found on examination ranging from general examination to systemic examinations. The data collected also included a detailed history, clinical examination and investigations with particular references to the hematological abnormalities. Data were presented by frequency and percentages and also by bar diagram. After compilation of data, the obtained data were checked, verified, edited and coded. The data were analyzed and statistical evaluation was performed by SPSS version 15.0 (Chicago, Illinois, USA) program. This study was approved by ethical review committee of Chittagong Medical College.

\section{RESULTS}

There were 40 patients included in the study with 38 (95\%) female and $2(5 \%)$ males. The most common presenting complaints were photosensitivity $(84.2 \%$ ) followed by fatigue $(50 \%)$ and rash $(45 \%)$ (Figure-1). All the patients satisfied at least 4 criteria needed for the diagnosis of SLE as per the recommendations. The various criteria are as showed in the figure- 2. Anemia was the most common hematological abnormality detected and was seen in 23(57.5\%) patients. Autoimmune hemolytic anemia(AIHA) was diagnosed in $2(8.7 \%)$ patients. $38(93.3 \%)$ patients had anemia due to various other causes including iron deficiency anemia( 60\%), and anemia of chronic diseases(ACD) 26\%. Fifty percent $(50 \%)$ patients had severe anemia where hemoglobin was $6.46 \pm 0.9 \mathrm{gm} \%$.(Table -1 and Table -2). Normocytic normochromic anemia(NNA) was the most common peripheral blood smear findings found in $20(50 \%)$ patients(Table-3). Leukocyte count was found $>4000 / \mathrm{cmm}$ in $33(82.5 \%)$ patients while leucopenia was found in $7(17.5 \%)$ (Table-4). Among the leucopenia Lymphopenia was predominant $(75 \%)($ Table -5$)$ Thrombocytopenia was found in $6(85 \%)$ patients(Table -6) A positive direct Coomb's test was found positive in $18(45.0 \%)$ patients and antiphospholipid antibody was assayed in 36 patients where 5( $13.9 \%)$ was found positive.(Table -7 and Table - 8)

Figure 1: Clinical features of the patients

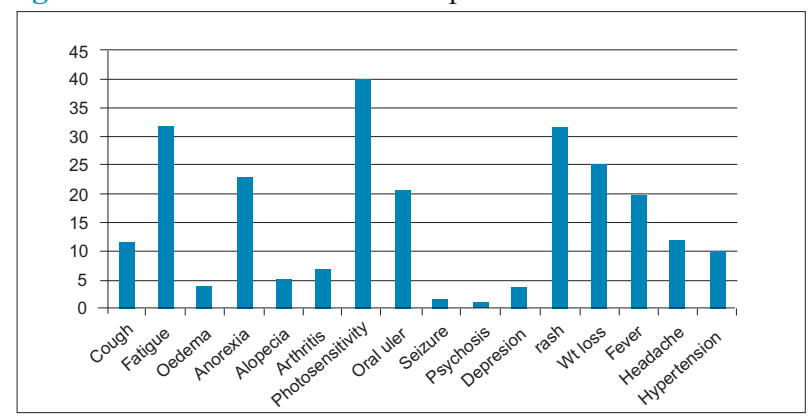

Figure 2: Diagnostic criteria of the patients

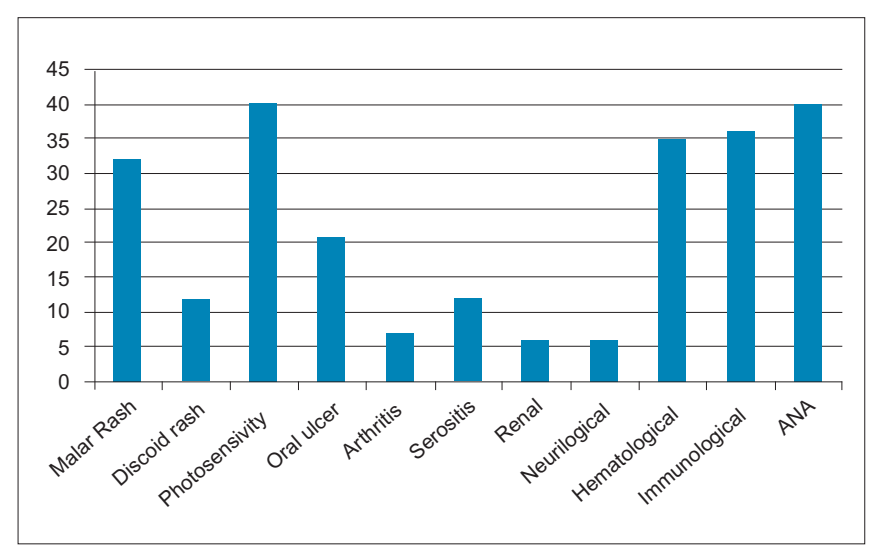

Table 1: Pattern of hematological abnormalities $(n=40)$

\begin{tabular}{lcc} 
Hematological abnormality* & Frequency & Percent \\
Anemia & 23 & 57.5 \\
Leucopenia & 7 & 17.5 \\
Thrombocytopenia & 3 & 7.5 \\
Pancytopenia & 2 & 5.0 \\
Normal & 5 & 12.5 \\
Total & 40 & 100.0 \\
\hline
\end{tabular}

* Diagnosed by $\mathrm{Hb} \%$ findings and PBF study

Table 2: Pattern of anemia of the patients $(n=40)$

\begin{tabular}{lcc} 
Pattern of Anemia* & Frequency & Percent \\
IDA** & 14 & 35.0 \\
ACD** & 6 & 15.0 \\
AIHA** & 2 & 5.0 \\
Others** & 1 & 2.5 \\
Total & 23 & 57.5 \\
No Anemia & 17 & 42.5 \\
Total & 40 & 100.0 \\
\hline
\end{tabular}

* Diagnosed by clinical parameter, PBF study and Coomb's test ** IDA= Iron Deficiency Anemia, ACD = Anemia of Chronic Disease, AIHA= Autoimmune Hemolytic Anemia

Table 3: Peripheral blood film findings $(n=40)$

\begin{tabular}{lcc} 
PBF findings & Frequency & Percent \\
NNA* & 12 & 30.0 \\
NHA* & 4 & 10.0 \\
MA* & 5 & 12.5 \\
Total & 21 & 55.0 \\
No anemia & 19 & 45.0 \\
Total & 40 & 100.0 \\
\hline
\end{tabular}

*NNA= Normocytic Normochromic Anemia, NHA= Normocytic Hypochromic Anemia, MA= Macrocytic Anemia 
Table 4: Pattern of Leukocyte abnormality $(n=40)$

\begin{tabular}{lcc} 
Leukocyte Abnormality* & Frequency & Percent \\
Lymphopenia & 6 & 15.0 \\
Neutropenia & 2 & 5.0 \\
Total & 8 & 20.0 \\
No Abnormality & 32 & 80.0 \\
Total & 40 & 100.0 \\
\hline
\end{tabular}

* Diagnosed by differential count analysis

Table 5: Pattern of Coombs test $(\mathrm{n}=40)$

$\begin{array}{ccc}\text { Coomb's Test* } & \text { Frequency } & \text { Percent } \\ \text { Positive } & 18 & 45.0 \\ \text { Negative } & 22 & 55.0 \\ \text { Total } & 40 & 100.0\end{array}$

Done by direct and indirect agglutination test

Table 6: Antiphospholipid Antibody $(n=40)$

\begin{tabular}{lcc} 
Anti phospholipid Ab Test* & Frequency & Percent \\
Positive & 10 & 25 \\
Negative & 26 & 75 \\
Total & 36 & 90.0 \\
Missing System & 4 & 10.0 \\
Total & 40 & 100.0 \\
\hline
\end{tabular}

*Done by immunological method

\section{DISCUSSION}

SLE is a chronic inflammatory disease with multisystem involvement having different clinical and immunological manifestation characterized by the presence of antinuclear antibodies. SLE most often harms the heart, joints, skin, lungs, blood vessels, liver, kidneys, and nervous system ${ }^{8}$. The course of the disease is unpredictable, with periods of illness alternating with remissions. The disease occurs nine times more often in women than in men, especially in women in child-bearing ages 15 to 35 , and is more common in those also of non-European descent.

This cross sectional study was done to evaluate the hematological manifestations among 40 patients with SLE. There was a female preponderance in the studied patients, females were $38(95 \%)$ and males $2(5 \%)$. Male female ratio was $1: 14$. This is an expected results as most of the studies ${ }^{8}$ show a similar results. Cameron et al has reported a male to female ratio of 1:8 to $1: 14$ in a series of adult patients ${ }^{7}$.

The mean age of the patients in our study was 28.42 years. SLE is a disease of child bearing age ${ }^{8}$. The median age of onset of $\mathrm{SLE}$ is 24 years in a series reported by Malaviya $\mathrm{AN}^{9}$.
The maximum period of duration of illness in our study was 612 months. In a study of a series of cases of 464 patients between 1980-1989, the mean duration of disease at diagnosis was 2.1 years $^{10}$. This trend is probably changing with the advent of newer diagnostic and serological tests. Lack of awareness among doctors, financial constrains of patients and the non availability of newer diagnostic tests in remote areas from where patients are referred to our hospital, would have contributed to the late diagnosis of the disease in our patients.

The most common presenting complaints were photosensitivity $(84.2 \%)$ followed by fatigue $(50 \%)$ and rash $(45 \%)$. Fever (44\%) was also a common presenting symptoms with arthralgias (41\%). But in some study done in India with a large sample size 321 the presenting problems were the arthralgias (76\%) and then the Fever $(61.9 \%)^{11}$. This difference from our study may be due to their large sample size and with some variation with life style and geographical variation.

Oral ulcer was found in $54.8 \%$ in our patients. Malviya[9] reports a $50 \%$ incidence of oral ulcers in his patients from eastern India. In a series of studies done by Wallace[10], oral ulcer were present in $7-36 \%$ of cases $^{10}$. Our study has documented a higher incidence of oral ulcers compared with western studies but the incidence is similar to that documented in the study from India. Malar rash was found in $45 \%$ of our patients. This observation also is similar to the series described by Wallace DJ where the incidence was $10-60 \%{ }^{10}$.

Overall the relative frequency of each of the major clinical features at presentations was similar to what has been documented in literature, except a much higher incidence of Photosensitivity in Bangladeshi patients as it is located near equatorial line.

The commonest criteria satisfied was ANA positivity. It was seen in all of our patients. This is an expected finding as ANA negative lupus is a rare clinical entity in our setting. ANA is the most sensitive indicator of the presence of SLE. ANA positivity is reported to in about $90-95 \%$ of cases $^{11}$.

Hematological abnormality were detected in $87.5 \%$ of patients diagnosed to have SLE in our study. In a study done in China by $\mathrm{Xu} \mathrm{XM}$ et al hematological abnormalities were detected in $86.2 \%$ of the patients ${ }^{12}$. Though there was such a high incidence of hematological features at presentation, none of these patients presented primarily with symptoms attributed to hematological abnormalities.

Most common hematological abnormality was anaemia $(57.5 \%)$. Various studies have shown a similar findings ${ }^{4}$. In a series of studies reviewed by BudMan anaemia occurred in 57$78 \%$ of patients with $\mathrm{SLE}^{4}$. Iron deficiency anemia, Anemia of chronic disease and Autoimmune hemolytic anemia are the common cause of anemia in SLE. Iron deficiency has been diagnosed on the basis of hypochromicity on the peripheral smear mainly, along with some clinical features like pica, nail changes, glossitis and chelitis. Anemia of chronic disease on the basis of a normocytic and normochromic blood picture in the presence of negative Coomb's test and AIHA on the basis of positive Coomb's test and reticulocytosis. Further investigations to evaluate anemia such as iron studies, serum folate levels and bone marrow examinations has not been done due to financial constraints. 
Iron deficiency anemia was the most common cause of anemia and accounted for $60 \%$ and anemia of chronic disease was $26.1 \%$. The findings is similar to that documented by Voulgarelis et $\mathrm{al}^{5}$.

A positive Coomb's test was observed in $45 \%$ of our patients but AIHA was documented in only $2(5 \%)$. Budman $\mathrm{R}$ in a review of a series of studies has found 18 to $65 \%$ of Coomb's positivity in patients with SLE. However the incidence of AIHA in his study was less than $10 \%{ }^{4}$.

Reticulocytosis was one of the diagnostic criteria for the diagnosis of AIHA. The bone marrow response to hemolysis in the form of reticulocytosis may be depressed if associated or folate deficiency is present or associated bone marrow suppression of chronic disease is present. This could be responsible for the gross discrepancy seen between the incidence of Coomb's positivity and the incidence of AIHA. More sophisticated test would perhaps have documented hemolysis in a greater number of patients.

Lymphopenia was predominant (75\%) among the WBC disorder. In a study by Debarre et al lymphopenia was detected in $84 \%$ of the cases and was found to be independent of leucopenia ${ }^{6}$. Thrombocytopenia was detected in $15 \%$ of patients but two of them had severe thrombocytopenia $<20,000 / \mathrm{cmm}$ at presentation.
None of them had any bleeding manifestations. Budman $\mathrm{R}$ describes an incidence of $14-26 \%$ thrombocytopenia in different series.

Antiphospholipid antibodies were detected in 10 subjects out of 36 patients $(26 \%)$ in whom screening was done. Anti phospholipid antibodies were detected in $12-30 \%$ of cases in a study which is similar to our study ${ }^{13}$.

Only three patients required blood transfusion. Though the incidence of anemia was very high in the study group, most of them responded to the treatment modalities given for primary underlying condition with DMARDs.

\section{CONCLUSION}

In conclusion it could be said that anemia is a common manifestations among the haematological manifestations of SLE patients. Iron deficiency anemia and positive Coomb's test are come important findings. This study is one center with small sample size so further study is warranted with large scale multi center design to find the national scenario.

\section{DISCLOSURE}

All the authors declared no competing interest. 


\section{REFERENCES}

1. James, William; Berger, Timothy; Elston, Dirk. Andrews' Diseases of the Skin: Clinical Dermatology. Saunders. 2005;10:345-456

2. Anisur Rahman and David A. Isenberg. "Review Article: Systemic Lupus Erythematosus". N Engl J Med 2008;358 (9): 929-939.

3. Hemminki K, Li X, Sundquist J, Sundquist K. "Familial associations of rheumatoid arthritis with autoimmune diseases and related conditions". Arthritis Rheum. 2009; 60 (3): 661-668.

4. Budman DR and Steinberg AD. Hematologic aspects of systemic lupus erythematosus. Current concepts. Ann Int Med 1977; 86: 220-229.

5. Voulgarelis M, Kokori SIG, Ioannidis JPA. Anemia in systemic lupus erythematosus: etiological profile and the role of erytropoetin . Ann Rheum Dis 2000;59: 217-222.

6. Miguel EC, Rodrigues Pereira RM, Debraganca Pereira CA. Psychiatric menefestations of systemic lupus erythematosus: Clinical features, symptoms and signs of central nervous system activity in 43 patients. Medicine1994;73:224-232.

7. Cameron SJ, Bank E, Melmerby . Lupus Nephritis. Disease of the month. J Am Soc Nephrol, 1999;10: 413-422.

8. Manzi M, Ramsay- Goldman, Star KV, Epidemiology of systemic lupus erythematosus. In Rheumatology, philadaelphia: Mosby. 2003;3:1295-1296.

9. Malaviya AN, Singh RR,Kumar A. Systemic lupus erythematosus in Northern India. A review of 329 cases. J Assoc. Physicians India. $1988 ; 36: 473-475$.

10. Wallace DJ, The clinical presentations of SLE, In Dubois' Lupus erythematosus, Wallace DJ and Hahn BH(eds) Philadelphia, Lea and Febiger 1993;4(33): 317-321.

11. Kumar A. Indian guideline on the management of SLE. Indian Rheumatologicalm Assoc 2002; 10: 80-96.

12. Hux M, Fan ZR, Zhou SY. Hematological abnormality and clinical characteristics in systemic lupus erythematosus. Zhongguo shi Yan. 2004;12(2):170-173.

13. Levin JS, Fritzler and Keith Elkon. Autoantibodies in SLE, In rheumatology, M.C. Hochberg, AJ Silman(eds) Philadelphia:Mosby 2003;3: 1337-1346. 